\title{
Marrying Wright's Law to Thermodynamics for a Relative Final Cost-predicting Model of Carbon-fuel Substitution
}

\author{
Roland H. Pawelke
}

\begin{abstract}
The problem of assessing the relative final cost of energy technology substitution is approached by means of a general interpretation of Wright's law and the introduction of the concept of thermodynamic utility which derives from energy carrier specific energy. Via the Bienaymé-Chebyshev inequality the ideal relative final cost of C-fuel substitution (diesel/gasoline) is estimated at three different probabilities (50\%, $87.5 \%$ and $96 \%$ ) for compressed hydrogen, liquid hydrogen, metal hydrides and Li-ion technology. C-fuel substitution at cost parity is an event clearly below the $50 \%$ probability horizon of insight, $96 \%$ values range between 1.8 ( $\mathrm{Li}$-ion) and 4.3 ( $\mathrm{LH} 2 / 350 \mathrm{bar}$ ) times the cost of C-fuel technology. The results are evinced in a cost/price comparison between the Toyota Mirai, Tesla 3 and Skoda Superb and found to reflect reality properly (see ESI). The approach also offers a systematic explanation for previously as-is reported findings of parameter studies from literature. The issue of mitigating the cost discrepancy is briefly discussed, too.
\end{abstract}

\section{Introduction}

The regularity between per-unit cost and manufacturing volume was described first by WRIGHT in 1936. ${ }^{1}$ He observed at U.S. aircraft manufacturer Curtiss-Wright that with each doubling of production numbers the per-unit labour cost receded by a constant proportion. This observation became known under the Learning Curve Effect or the more catchy term "Wright's law". While the effect is solid, the causality at work is far less so: e.g. the Boston Consulting Group later extended the scope towards process experience: ${ }^{2}$ In sum, it is an empiric law. The power function of equation 1 shows its mathematics: $P_{x}$ being the per-unit price at cumulative quantity $x, P_{1}$ the initial per-unit price and $b$ the empiric factor, usually $0.75<b<0.90,(1-b)$ gives the proportion by which the perunit cost is reduced. Hence, the most general interpretation of Wright's law possible is a logarithmic factor indicating the advantage to per-unit cost in relation to cumulative production amount.

$P_{\mathrm{x}}=P_{1} x^{\log _{2}(b)}$

The model might appear simple but yielded the best overall result in a comparative statistical analysis of six other technology-cost forecasting models published in 2013 by NAGY et al, even ahead of the vaunted Moore's law. ${ }^{3}$ The study was done across 62 different technologies and the effect is prevalent across the board although at a variety in emphasis. Interestingly, knowledge of the actual causality per se is not vital and if scope allows for it, adopting an "agnostic view regarding the causal mechanism" may not only be admissible but expedient. ${ }^{4}$ NAGY et al emphasize the evident difficulty if not impossibility of condensing the manufacture of a complex item such as a car or airplane into a single coefficient but it is worth questioning whether this also applies to energy technologies. On a superficial glance the answer appears to be clearly "Yes" if only for the complexity of the item; but if thought through to its ideal conclusion, the answer actually becomes "No": Energy technologies are different since tied to thermodynamics which very much benefits a single-digit reduction of the kind.

\section{Methodical Approach}

Quantity $b$ in equation 1 may be interpreted as an advantage factor whose deeper causality needs not to be known. In an ideal case, i.e. unlimited access to labour and materials in a free market with demand always slightly trailing ahead of supply, the difference in cost between two energy technologies must be ultimately vindicated by a disparity in thermodynamic utility, i.e. the specific 
energy of the energy carrier of which a certain proportion is made available. Equation 2 shows that the specific energy of an energy carrier may be expressed as pressure $p$ per medium density $\rho$ which is uncommon but valid, making it possible to compare e.g. a gaseous energy carrier with a liquid one.

$1 \mathrm{Wh} \mathrm{kg}^{-1}=36$ bar $\mathrm{L} \mathrm{kg}^{-1}=p \rho^{-1} \Rightarrow$ advantage factor $b=\frac{\text { specific energy }\left[\mathrm{Lbar} \mathrm{kg}^{-1}\right] \text { density } \rho\left[\mathrm{kg} \mathrm{L}^{-1}\right]}{\text { pressure } p \text { [bar }]}$

Hence the specific energy of an energy carrier can be broken down into a single dimensionless figure by relating it to the pressure and medium density of the storage state. The crucial role of the storage state is for the reason it represents in terms of effort the apex of an energy carrier utilization chain: it has been produced or mined, purified and filled into the tank: figuratively speaking, it is downhill either to the well or the wheel. That dimensionless number derived from the specific energy may be seen a base measure for said thermodynamic advantage $b$. This quantity $b$ has little meaning in its own right but if expressed a) as natural logarithm and b) put in relation to Wright's law in the sense of a utility factor, the ultimate relative difference in cost between two energy technology options will be given by the quotient of both utility factors in the above sense. Towards that end, equation 1 is transformed for the example of a generic technology option $(P, x, b)$ as shown in equation 3.

$P_{x}=P_{1} x^{\log _{2}(b)} \Rightarrow \quad \ln \left(\frac{P_{x}}{P_{1}}\right)=\ln (x) \log _{2}(b) \quad \Rightarrow \quad \ln \left(\frac{P_{x}}{P_{1}}\right) \frac{\ln (2)}{\ln (x)}=\ln (b)$

Equation 3 is the fundamental expression for the per-unit cost development and equation 4 shows an exemplary relative cost comparison between two options $(P, x, b)$ and $(Q, x, c)$.

$\frac{\ln (b)}{\ln (c)}=\frac{\ln (2) \ln (x)}{\ln (2) \ln (x)} \frac{\ln \left(\frac{P_{x}}{P_{1}}\right)}{\ln \left(\frac{Q_{x}}{Q_{1}}\right)}$

Equation 4 highlights that Wright's law greatly simplifies in a comparison at equal production amount $x$ : the issue breaks then down to determining the utility values $\ln (b)$ and $\ln (c)$; knowledge of the actual cost quantities on equation's 4 right side is not vital. However, equation 4 needs further refinement as only a fraction of the tank's specific energy is made effective; towards that end the relative efficiency factors $\beta$ and $\gamma$ are introduced to $b$ and $c$, respectively; shown in equation 5 .

$\frac{\ln (\beta b)}{\ln (\gamma c)}=\frac{\left(\frac{\ln (\beta)}{\ln (b)}+1\right) \ln (b)}{\left(\frac{\ln (\gamma)}{\ln (c)}+1\right) \ln (c)}$

The issue of how to figure the effect of the unknown $\beta$ and $\gamma$ coefficients may be dealt with by a statistical approach of symmetry: since efficiency factors $\beta$ and $\gamma$ are always between 0 and 1 , it is sensible to define $\beta$ and $\gamma$ each by the value pair of 0.25 and 0.75 yielding a mean of 0.5 with $\Delta \beta_{\max }=$ $\Delta \gamma_{\max }=0.25$; hence the distance between mean and ultimate interval borders is equal. Since the quotient of logarithms in equation 5 relates linearly, the interval $[0.25,0.75]$ is by amount also a measure for the interval $[0.5,0]$ respective $[0.5,1]$. The problem then simplifies to assessing the deviation from the quotient of $\ln (b)$ and $\ln (c)$ at $\beta=\gamma=0.5$ whose expected value $\mathrm{E}(\mathrm{X})$ is shown in equation $6 \mathrm{a}$; the regularity $\mathrm{F}(\mathrm{X})$ by which said deviation occurs is defined by equation $6 \mathrm{~b}$; the variance is given by the permutations of $\beta$ and $\gamma$ as shown in equation $6 c$. 


$$
\begin{aligned}
& \mathrm{E}(\mathrm{X}):=\frac{\left(\frac{\ln (0.5)}{\ln (b)}+1\right)}{\left(\frac{\ln (0.5)}{\ln (c)}+1\right) \frac{\ln (b)}{\ln (c)}} \quad \beta, \gamma \in\{0.25,0.75\} \\
& \mathrm{F}(\mathrm{X}):=\frac{\ln (\beta b)}{\ln (\gamma c)}=\frac{\left(\frac{\ln (\beta)}{\ln (b)}+1\right) \ln (b)}{\left(\frac{\ln (\gamma)}{\ln (c)}+1\right) \ln (c)} \quad\left(\frac{\left(\frac{\ln (0.5)}{\ln (b)}+1\right)}{\left(\frac{\ln (0.5)}{\ln (c)}+1\right)} \frac{\ln (b)}{\ln (c)}-\frac{\left(\frac{\ln (0.25)}{\ln (b)}+1\right)}{\left(\frac{\ln (0.25)}{\ln (c)}+1\right)} \frac{\ln (b)}{\ln (c)}\right)^{2}+\left(\frac{\left(\frac{\ln (0.5)}{\ln (b)}+1\right)}{\left(\frac{\ln (0.5)}{\ln (c)}+1\right)} \frac{\ln (b)}{\ln (c)}-\frac{\left(\frac{\ln (0.25)}{\ln (b)}+1\right)}{\left(\frac{\ln (0.75)}{\ln (c)}+1\right)} \frac{\ln (b)}{\ln (c)}\right)^{2}+ \\
& \operatorname{Var}(\mathrm{X})=\sigma^{2}+ \\
& \quad\left(\frac{\left(\frac{\ln (0.5)}{\ln (b)}+1\right)}{\left(\frac{\ln (0.5)}{\ln (c)}+1\right)} \frac{\ln (b)}{\ln (c)}-\frac{\left(\frac{\ln (0.75)}{\ln (b)}+1\right)}{\left(\frac{\ln (0.25)}{\ln (c)}+1\right)} \frac{\ln (b)}{\ln (c)}\right)^{2}+\left(\frac{\left(\frac{\ln (0.5)}{\ln (b)}+1\right)}{\left(\frac{\ln (0.5)}{\ln (c)}+1\right)} \frac{\ln (b)}{\ln (c)}-\frac{\left(\frac{\ln (0.75)}{\ln (b)}+1\right)}{\left(\frac{\ln (0.75)}{\ln (c)}+1\right)} \frac{\ln (b)}{\ln (c)}\right)^{2}
\end{aligned}
$$

Based on equations $6 a$ and $6 c$ it is possible to determine via the Bienaymé-Chebyshev inequality the interval from $E(X)$ in which random values can be found at a certain minimum probability. The factors $\beta$ and $\gamma$ are not exactly random quantities but since they may show a broad independent variety, random is a viable approximation. Three cases are selected: the horizon of insight lies at a probability of 50:50, a low probability case at 87.5:12.5 and a high probability case at 96.0:4.0. The former is given by $\sqrt{2} \sigma$, the others by $2 \sqrt{2} \sigma$ and $5 \sigma$, respectively. This $5 \sigma$ limit is sensible because it aligns the trade-off between interval size and probability with the general quality rule of statistics that a confidence interval should in $95 \%$ of all cases enclose the unknown true value. The values $\mathbb{E}_{50.0}, \mathbb{E}_{87.5}$ and $\mathbb{E}_{96.0}$ are obtained as by equations 7 a to $7 c$ (only addition is of practical sensibility).

$$
\begin{aligned}
& \mathbb{E}_{50.0}\left(\frac{\ln (\beta b)}{\ln (\gamma c)}\right)=\frac{\left(\frac{\ln (0.5)}{\ln (b)}+1\right)}{\left(\frac{\ln (0.5)}{\ln (c)}+1\right)} \frac{\ln (b)}{\ln (c)}+\sqrt{2} \sigma \\
& \mathbb{E}_{87.5}\left(\frac{\ln (\beta b)}{\ln (\gamma c)}\right)=\frac{\left(\frac{\ln (0.5)}{\ln (b)}+1\right)}{\left(\frac{\ln (0.5)}{\ln (c)}+1\right)} \frac{\ln (b)}{\ln (c)}+2 \sqrt{2} \sigma \\
& \mathbb{E}_{96.0}\left(\frac{\ln (\beta b)}{\ln (\gamma c)}\right)=\frac{\left(\frac{\ln (0.5)}{\ln (b)}+1\right)}{\left(\frac{\ln (0.5)}{\ln (c)}+1\right)} \frac{\ln (b)}{\ln (c)}+5 \sigma
\end{aligned}
$$

The issue of unknown efficiency coefficients $\beta$ and $\gamma$ can be thus mitigated without need of actual knowledge of them; even the comparatively weak bound prediction capability of the BienayméChebyshev is in this context rather an advantage considering the unspecified possible multitude of elastic sub-factors which may contribute to coefficients $\beta$ and $\gamma$.

\section{Results}

\subsection{Proof of concept on basis of a crude base model}

The thermodynamic advantage and utility coefficients according to equation 2 are determined for seven energy carrier utilization modes: diesel fuel, motor gasoline, compressed hydrogen $(\mathrm{cH} 2)$ at 350 bar and 700 bar, light metal hydride $\left(\mathrm{LMH} \text {, based on }\left[\mathrm{AlH}_{4}\right] \text {-stage of } \mathrm{K} / \mathrm{Ti} \text {-doped } \mathrm{NaAlH}_{4}\right)_{1}^{5,6}$ heavy 
metal hydride $(\mathrm{HMH}$, based on Hydralloy $\mathrm{C5}){ }^{7,8}$ liquefied hydrogen at 1 bar ( $\left.\mathrm{LH} 2\right)$ and lithium-ion cell (Li-ion). The specific energy of the carbon-fuels is derived on basis of the tonne of oil equivalent (toe) conversion system as by the International Energy Agency: a toe has the energy equivalent of 11.63 MWh. All densities are the values for the plain energy carriers. Table 1 displays the results.

Table 1 Determination of utility coefficients for various energy storage technologies.

\begin{tabular}{|c|c|c|c|c|c|c|}
\hline & \multicolumn{2}{|c|}{ Specific energy } & \multirow{2}{*}{$\begin{array}{c}\text { Storage Pressure } p \\
\text { [bar] }\end{array}$} & \multirow{2}{*}{$\begin{array}{c}\text { Density } \rho \\
{\left[\mathrm{kg} \mathrm{L}^{-1}\right]}\end{array}$} & \multirow{2}{*}{$\begin{array}{l}\text { Advantage } \\
\text { factor } b\end{array}$} & \multirow{2}{*}{$\begin{array}{l}\text { Utility } \\
\ln (b)\end{array}$} \\
\hline & {$\left[\mathrm{kWh} \mathrm{kg}^{-1}\right]$} & {$\left[\mathrm{kWh} \mathrm{kg}^{-1}\right]$} & & & & \\
\hline Diesel fuel $^{\mathrm{a}}$ & 11.24 & 404640 & 1 & 0.840 & 339897.6 & 12.7364 \\
\hline Motor gasoline ${ }^{b}$ & 10.87 & 391320 & 1 & 0.745 & 291533.4 & 12.5829 \\
\hline cH2 (350 bar, $288 \mathrm{~K})$ & 33.33 & 1199880 & 350 & 0.024 & 82.3 & 4.4104 \\
\hline cH2 (700 bar, 288 K) & 33.33 & 1199880 & 700 & 0.040 & 68.6 & 4.2283 \\
\hline LH2 (1 bar, 20 K) & 33.33 & 1199880 & 1 & 0.071 & 85191.5 & 11.3527 \\
\hline LMH & $\begin{array}{c}0.933 \\
(2.8 \% \mathrm{w} / \mathrm{w} \mathrm{H}) \\
\end{array}$ & 33588 & 80 & 1.270 & 533.2 & 6.2789 \\
\hline HMH & $\begin{array}{c}0.533 \\
(1.6 \% \mathrm{w} / \mathrm{w} \mathrm{H}) \\
\end{array}$ & 19188 & 15 & 5.595 & 7157.1 & 8.8759 \\
\hline Li-ion $^{c}$ & 0.224 & 8064 & 1 & 3.094 & 24950.0 & 10.1246 \\
\hline
\end{tabular}

\subsection{Refinement of the base model}

The figures of table 1 reveal three noteworthy findings: First, the difference between Diesel and Motor Gasoline logarithmic advantage factors is not very pronounced; the same is applies for both compressed hydrogen cases due to the relation between pressure and density. The latter finding is in accordance with a well-to wheel efficiency parameter study from $2005 .{ }^{9}$ Consequently, both cases may be subsumed as $\mathrm{C}$-fuel and $\mathrm{cH} 2$, respectively. Second, despite hydrogen packing a high nominal specific energy, the actual utility of compressed gas storage emerges last! This is due to a combination of high pressure and low medium density: within the concept of thermodynamic utility, specific energy is important but storage pressure and medium density can easily offset the benefits of an apparent high specific energy. That works also the other way as the Li-ion and metal hydrides cases show. In sum, this pins down the reason for why a sole focus on specific energy or gravimetric reversible hydrogen storage capacity (for metal hydrides) falls too short of aim. Third, all hydrogenrelated figures of table 1 are generally too high because there is a temperature-dependent factor to pressure $p$ in equation 2: the effect had been tacitly sidelined far for the sake of straightforwardness in the line of argument but merits now recognition. The coefficient of thermal volume expansion $\alpha_{v}$ is hidden in the volume part of the density as outlined in equation 8.

Advantage factor $b=p \rho^{-1}=p \frac{V}{m}=p \frac{V\left(1+\alpha_{v} \Delta T\right)}{m} \Rightarrow p^{\prime}=p\left(1+\alpha_{v} \Delta T\right) \quad \Rightarrow \quad b=p^{\prime} \frac{V}{m}$

Equation 8 shows that within the concept of thermodynamic advantage a temperature spread $\Delta T$ vital to energy carrier storage has a reducing effect. This reduction can be accounted for either by means of an abatement on specific energy or by increasing the nominal storage pressure $p$ to $p^{\prime}$ as seen in equation 8 . Coefficient $\alpha_{v}$ ranges by orders of magnitude from about $10^{-7} \mathrm{~K}^{-1}$ for hard solids to about $10^{-3} \mathrm{~K}^{-1}$ for organic liquids. The effect of thermal energy carrier volume expansion may be neglected for $\mathrm{C}$-fuel and Li-ion energy storage because of the small $\Delta T$ and the logarithmic form of utility. However, due to the pronounced relation between gas volume, pressure and temperature, this effect needs to be taken into account for hydrogen technologies; all the more temperature is a means to modulate the $\mathrm{LH} 2$ and metal hydride hydrogen storage processes. The thermal volume 
expansion coefficient of hydrogen is assumed as constant and near-ideal with $\alpha_{v}=(273.07 \mathrm{~K})^{-1}=$ $36.62 \cdot 10^{-4} \mathrm{~K}^{-1}$.

With regard to $\mathrm{cH} 2$ storage in type IV tanks, ${ }^{10}$ the vessel temperature should remain below $85^{\circ} \mathrm{C}$ for safety reasons due to the issue of de-lamination which marks the upper limit to $\Delta T$. The lower limit is given by fuelling protocol SAE TIR J2601: there only two dispenser types for 700 bar storage which require pre-cooling to either $-20^{\circ} \mathrm{C}$ or $-40{ }^{\circ} \mathrm{C} ;{ }^{11}$ those temperatures apply for 350 bar dispensers as well. While for 350 bar delivery pressure there are also dispenser types operating at a pre-cooling temperature of $0{ }^{\circ} \mathrm{C}$ or even ambient temperature, $\mathrm{CH} 2$ storage is now an average consideration on the logarithmic utility scale. For that reason the dispenser operating at $-20{ }^{\circ} \mathrm{C}$ is considered, corresponding to a $\Delta T$ of $105^{\circ} \mathrm{C}$ : this equals an increase of $38.44 \%$ or $p^{\prime}$ values of 969 bar and 485 bar, respectively. These values may be understood as the peak pressures of 875 bar and 438 bar as by SAE J2601 hydrogen fuelling protocol approach plus a $10.7 \%$ or $3.6 \mathrm{kWh}\left(\mathrm{kg} \mathrm{H}_{2}\right)^{-1}$ penalty on utility for the pre-cooling/compression process. ${ }^{12}$

Metal hydride hydrogen storage is insofar different from that because storage is bound to a phase transition reverting which consumes heat. In order to capture that relation, the pressure shift $\Delta p$ due to a temperature change $\Delta T$ (at constant molar volume $V_{\mathrm{m}}$ ) from an (known) initial pressure $p$ and temperature $T$ is expressed by the ideal gas law as by equation $9 a$.

$\frac{\Delta p V_{\mathrm{m}}}{p V_{\mathrm{m}}}=\frac{\Delta T \mathrm{R}}{T \mathrm{R}} \Rightarrow \frac{\Delta p}{p}=\frac{\Delta T}{T} \quad \Rightarrow \quad p=\frac{\Delta p}{\Delta T} T$

Inserting the expression for $p$ of equation 9a into equation 8 yields equation 9b:

$p^{\prime}=p\left(1+\alpha_{v} \Delta T\right) \quad \Rightarrow \quad p^{\prime}=\frac{\Delta p}{\Delta T} T\left(1+\alpha_{v} \Delta T\right) \quad \Rightarrow \quad p^{\prime}=\Delta p\left(\frac{T}{\Delta T}+\alpha_{v} T\right)$

In order to advance from equation $9 \mathrm{~b}$ a notion from the practice of metal hydride fuel cell energy system development is due: it is not sufficient just to match the equilibrium pressure above the metal hydride with the hydrogen supply pressure of the fuel cell. For a steady supply respective a contingency reservoir the equilibrium pressure must be higher which depends on fuel cell type, metal hydride kinetics and the application's load profile. However, a metal hydride equilibrium pressure three times the actual fuel cell operating pressure may serve as reference and the line of argument will develop along that example. The spread $\Delta p$ is thus defined by difference $\left(p^{*}-p\right)$ with $p^{*}=p_{\text {eq }}=3 p$, on the basis of the known initial pressure $p$ (for fuel cell supply). The pressure shift $\Delta p$ is expressed by means of van't Hoff terms $(\Delta H>0)$ for the temperature spread $\Delta T$, see equation 10a.

$\ln \left(\frac{p^{*}}{p^{\circ}}\right)-\ln \left(\frac{p}{p^{\circ}}\right)=\frac{-\Delta H}{\mathrm{R} T^{*}}+\frac{\Delta S}{\mathrm{R}}-\left(\frac{-\Delta H}{\mathrm{R} T}+\frac{\Delta S}{\mathrm{R}}\right) \quad \Rightarrow \quad \ln \left(\frac{p^{*}}{p}\right)=\frac{\Delta H}{\mathrm{R}}\left(\frac{1}{T}-\frac{1}{T^{*}}\right)$

Substituting $p^{*}=3 p$ into equation $10 \mathrm{a}$ and solving for $T^{*}$ yields equation $10 \mathrm{~b}$ :

$\ln (3)=\frac{\Delta H}{\mathrm{R}}\left(\frac{1}{T}-\frac{1}{T^{*}}\right) \Rightarrow \frac{1}{T^{*}}=\frac{1}{T}-\frac{\mathrm{R} \ln (3)}{\Delta H}=\frac{\Delta H-\mathrm{R} T \ln (3)}{\Delta H T} \Rightarrow T^{*}=\frac{\Delta H T}{\Delta H-\mathrm{R} T \ln (3)}$

Insertion of equation $10 \mathrm{~b}$ into equation $9 \mathrm{~b}$ with $\Delta p=\left(p^{*}-p\right)$ with $p^{*}=3 p$ and $\Delta T=\left(T^{*}-T\right)$ yields:

$\Delta T=\left(T^{*}-T\right)=\frac{\Delta H T}{\Delta H-\mathrm{R} T \ln (3)}-T=T\left(\frac{\Delta H}{\Delta H-\mathrm{R} T \ln (3)}-1\right)$ 
$p^{\prime}=(3 p-p)\left(\frac{T}{\Delta T}+\alpha_{v} T\right) \Rightarrow \quad p^{\prime}=2 p\left(\left(\frac{\Delta H}{\Delta H-\mathrm{R} T \ln (3)}-1\right)^{-1}+\alpha_{v} T\right)$

(10c continued)

Equation $10 \mathrm{c}$ is the sought expression for assessing the impact of temperature on utility for a phasechange storage method such as metal hydrides or LH2. Equation 11 is a generalization of $10 \mathrm{c}$ for any ratio $n$ of metal hydride equilibrium to fuel cell pressure $p ; n \cdot p$ equals the desired reservoir pressure.

$p^{\prime}=(n-1) p\left(\left(\frac{\Delta H}{\Delta H-\mathrm{R} T \ln (n)}-1\right)^{-1}+\alpha_{v} T\right)$

Equation 11 may be also applied to LH2 storage with a heat of evaporation of $912 \mathrm{~J}\left(\mathrm{~mol} \mathrm{H}_{2}\right)^{-1}$ in lieu of desorption enthalpy $\Delta H$. The LH2 values in table 1 are considerably too high: whereas for $\mathrm{CH} 2$ hydrogen storage the penalty for compression on utility could be naturally accounted for by means of pressure $p^{\prime}$, it is more straightforward for LH2 storage to place the penalty on specific energy from the start and reduce it by the about $11.9 \mathrm{kWh}\left(\mathrm{kg} \mathrm{H}_{2}\right)^{-1}$ the liquefaction process consumes. ${ }^{13}$ There is furthermore the issue of boil-off loss aka dormancy issue, a quite elastic quantity since depending much on externalities such as scale and pressure, e.g. a 350 bar cryo-tank has been used to avoid the issue altogether. ${ }^{14}$ An approximate solution for the issue is modelling the boil-off loss on the basis of data by PETITPAS. ${ }^{15}$ However, the initial data form "percentage lost per kg-amount LH2" transferred is differently expressed for "residual LH2 watt-day(s)" (since kg LH2/day $=$ Wh $\left(24 \mathrm{~h} /\right.$ day) ${ }^{-1}=$ watt-day). Relating this to a 8 watt-day dormancy reference for standard LH2 cryo-storage vessels eliminates time as a factor; ${ }^{12}$ scaling effects are removed by polynomial fitting (see ESI): the zero-order term figures the invariant penalty to $40 \%$. Thus, in the LH2 case specific energy recedes from $33.33 \mathrm{kWh}$ $\mathrm{kg}^{-1}$ to about $0.6(33.33-11.9) \mathrm{kWh} \mathrm{kg}^{-1}=12.86 \mathrm{kWh} \mathrm{kg}^{-1}$.

PEMFCs operate at pressures above 2 bar for (quasi-)stationary purposes; applications of a more dynamic profile such as e.g. fuel cell electric vehicles (FCEV) require a reservoir pressure between 10 to 15 bar as by the Toyota Mirai fuel cell vehicle dismantling manual. This spread is accounted for by setting $p=2$ bar and $n=7.5$ in equation 11 . This yields for LH2 a pressure $p^{\prime}$ according to equation 11 of 22 bar. Since the use of LH 2 in combination with 350 bar tanks has been described as a mitigation strategy for the dormancy issue, ${ }^{12}$ it is also taken into consideration. With regards to metal hydrides, densities are adjusted for an about poured random packing $(62 \%) .{ }^{16}$ The $p^{\prime}$ pressure values are calculated as by equation 11 but for $\mathrm{K} / \mathrm{Ti}$-doped $\mathrm{NaAlH}_{4}$ the (higher) pressure of 79 bar is retained with regard to practical hydrogenation speed. ${ }^{5}$ Furthermore, a prospective light metal hydride $\mathrm{LMH}^{*}$ is added, estimated figures base on $T=453^{\circ} \mathrm{C}$ and $\Delta H=46 \mathrm{~kJ}(\mathrm{~mol} \mathrm{H}) .{ }^{17}$ Table 2 shows the results.

Table 2 Refined table 1 data with regard to abatements on utility.

\begin{tabular}{|c|c|c|c|c|c|c|c|}
\hline & \multicolumn{2}{|c|}{ Specific energy } & \multirow{2}{*}{$\begin{array}{l}\text { Storage Pressure } \\
p^{\prime}[\text { bar] }\end{array}$} & \multirow{2}{*}{$\begin{array}{c}\text { Density } \rho \\
{\left[\mathrm{kg} \mathrm{L}^{-1}\right]}\end{array}$} & \multirow{2}{*}{$\begin{array}{l}\text { Advantage } \\
\text { factor } b\end{array}$} & \multirow{2}{*}{\multicolumn{2}{|c|}{ Utility $\ln (b)$}} \\
\hline & {$\left[\mathrm{kWh} \mathrm{kg}^{-1}\right]$} & {$\left[\mathrm{L} \mathrm{bar} \mathrm{kg}^{-1}\right]$} & & & & & \\
\hline C-fuel & - & - & - & - & - & & 597 \\
\hline cH2 (288 K, 350 bar) & 33.33 & 1199880 & 485 & 0.024 & 59.4 & 4.0839 & \\
\hline cH2 (288 K, 700 bar) & 33.33 & 1199880 & 969 & 0.040 & 49.5 & 3.9026 & \\
\hline 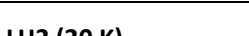 & 1286 & 1962060 & 22 & 0071 & 1494.1 & & \\
\hline $\operatorname{Li} 2(20 \mathrm{~K})$ & 12.86 & 462960 & 350 & $0.0 / 1$ & 93.9 & & \\
\hline LMH (K/Ti-NaAlH 4 ) & $0.933(2.8 \%$ w/w H) & 33588 & 79 & 0.787 & 334.6 & & \\
\hline LMH* (prospective) & $1.267(3.8 \% \mathrm{w} / \mathrm{w} \mathrm{H})$ & 45612 & 37 & 1.372 & 1691.3 & & \\
\hline HMH (Hydralloy C5) & $0.533(1.6 \% \mathrm{w} / \mathrm{w} \mathrm{H})$ & 19188 & 34 & 3.469 & 1957.7 & & \\
\hline Li-ion & 0.224 & 8064 & 1 & 3.094 & 24950.0 & & 246 \\
\hline
\end{tabular}


On the basis of table 2 figures with equations $6 c$ and $7 a$ to $7 c$ the $\mathbb{E}_{50.0}, \mathbb{E}_{87.5}$ and $\mathbb{E}_{96.0}$ values for the ratio of final utility respective cost are determined (the relation is assumed reciprocal), see table 3 .

Table 3 Assessment of the relative final cost for C-fuel substitution on basis of table 2 figures.

\begin{tabular}{|c|c|c|c|c|c|c|c|c|}
\hline & C-fuel & $\mathrm{cH} 2$ & $\begin{array}{c}\text { LH2 } \\
\text { (22 bar) }\end{array}$ & $\begin{array}{c}\text { LH2 } \\
\text { (350 bar) }\end{array}$ & LMH & LMH* & $\mathrm{HMH}$ & Li-ion \\
\hline Utility factor $\ln (b)$ & 12.6597 & 3.9933 & 7.3093 & 3.6863 & 5.8129 & 7.4333 & 7.5795 & 10.1246 \\
\hline$E(X)$ (equation $6 a)^{d}$ & 1.0000 & 1.1438 & 1.0443 & 1.1641 & 1.0731 & 1.0425 & 1.0404 & 1.0147 \\
\hline $\begin{array}{l}\text { Standard deviation } \sigma \\
\text { of } E(X)\end{array}$ & - & 0.5308 & 0.2181 & 0.6172 & 0.2915 & 0.2139 & 0.2092 & 0.1567 \\
\hline $\begin{array}{l}\mathbb{E}_{50.0} \text { relative cost to } \\
\text { C-Fuel, } \sqrt{ } 2 \sigma+E(X)\end{array}$ & - & $\begin{array}{c}1.8944 \approx \\
1.9\end{array}$ & $\begin{array}{c}1.3527 \approx \\
1.4\end{array}$ & $\begin{array}{c}2.0370 \approx \\
2.0\end{array}$ & $\begin{array}{c}1.4854 \approx \\
1.5\end{array}$ & $\begin{array}{c}1.3450 \approx \\
1.3\end{array}$ & $\begin{array}{c}1.3363 \approx \\
1.3\end{array}$ & $\begin{array}{c}1.2363 \approx \\
1.2\end{array}$ \\
\hline $\begin{array}{l}\mathbb{E}_{87.5} \text { relative cost to } \\
\text { C-fuel, } 2 \sqrt{2} \sigma+E(X)\end{array}$ & - & $\begin{array}{c}2.6451 \approx \\
2.7\end{array}$ & $\begin{array}{c}1.6612 \approx \\
1.7\end{array}$ & $\begin{array}{c}2.9099 \approx \\
2.9\end{array}$ & $\begin{array}{c}1.8977 \approx \\
1.9\end{array}$ & $\begin{array}{c}1.6475 \approx \\
1.6\end{array}$ & $\begin{array}{c}1.6322 \approx \\
1.6\end{array}$ & $\begin{array}{c}1.4578 \approx \\
1.5\end{array}$ \\
\hline $\begin{array}{l}\mathbb{E}_{96.0} \text { relative cost to } \\
\text { C-fuel, } 5 \sigma+E(X)\end{array}$ & - & $\begin{array}{c}3.7978 \approx \\
3.8\end{array}$ & $\begin{array}{c}2.1349 \approx \\
2.1\end{array}$ & $\begin{array}{c}4.2503 \approx \\
4.3\end{array}$ & $\begin{array}{c}2.5308 \approx \\
2.5\end{array}$ & $\begin{array}{c}2.1121 \approx \\
2.1\end{array}$ & $\begin{array}{c}2.0865 \approx \\
2.1\end{array}$ & $\begin{array}{c}1.7981 \approx \\
1.8\end{array}$ \\
\hline
\end{tabular}

${ }^{d}$ For utility and cost relate reciprocally in the model, C-fuel in the nominator yields instantly the relative cost factor.

Table 3 show the ideal final cost ratios within terms and conditions of the approach: substitution of C-fuels at cost-parity is an event clearly below the $50 \%$ insight horizon of the model. The question arises what could be possible boundary conditions at which cost parity might ensue which will be treated with on basis of the $\mathbb{E}_{96.0}$ values. Table 3 figures rest on the premise of equal production amount, hence a cost disparity might be absorbed by production numbers: the relative amount can be estimated on the basis of equations 3 and 4 and $\mathbb{E}_{96.0}$ values of table 3 . Equations 12 show the factor $y$ by which the cumulative amount $x$ needs to scale for cost parity (via auxiliary variable $j$ ).

$\left(\frac{\ln (\beta b)}{\ln (\gamma c)}\right) \frac{\ln (x)}{\ln (x)}=j=\mathbb{E}_{96.0}\left(\frac{\ln (\beta b)}{\ln (\gamma c)}\right) \quad$ for $\mathbb{E}_{96.0}\left(\frac{\ln (\beta b)}{\ln (\gamma c)}\right)=1 \quad \Rightarrow \quad \frac{\ln (x)}{\ln (x)+\ln (y)}=\frac{1}{j}=\frac{1}{\mathbb{E}_{96.0}}(\mathbf{1 2 a})$

$1+\frac{\ln (y)}{\ln (x)}=j=\mathbb{E}_{96.0} \quad \Rightarrow \quad \frac{\ln (y)}{\ln (x)}=j-1=\mathbb{E}_{96.0}-1 \quad \Rightarrow \quad \frac{y}{x}=e^{(j-1)}$

Equation $12 \mathrm{~b}$ with $\mathbb{E}_{96.0}$ values from table 3 yields the relative production ratios as shown in table 4 .

Table 4 Relative production ratio for absorbing relative final cost disparity on basis of $\mathbb{E}_{96.0}$ values from table 3 .

\begin{tabular}{|l|c|c|c|c|c|c|c|}
\hline & $\mathbf{c H 2}$ & $\begin{array}{c}\text { LH2 } \\
\mathbf{2 2} \text { bar) }\end{array}$ & $\begin{array}{c}\text { LH2 } \\
\mathbf{3 5 0} \text { bar) }\end{array}$ & LMH & LMH* & HMH & Li-ion \\
\hline $\begin{array}{l}\mathbb{E}_{96.0} \text { value of relative } \\
\text { cost to C-fuel }\end{array}$ & 3.8 & 2.1 & 4.3 & 2.5 & 2.1 & 2.1 & 1.8 \\
\hline $\begin{array}{l}\text { Ratio y:x required for } \\
\text { C-fuel cost parity }\end{array}$ & 16.4446 & 3.0042 & 27.1126 & 4.4817 & 3.0042 & 3.0042 & 2.2255 \\
\hline $\begin{array}{l}\text { Relative market share } \\
\text { to C-fuel for cost parity }\end{array}$ & $94 \%$ & $67 \%$ & $96 \%$ & $78 \%$ & $67 \%$ & $67 \%$ & $55 \%$ \\
\hline
\end{tabular}

Table 4 shows what might be termed a planned economy scenario, highlighting the minimum market share required for a C-fuel substitution technology in order to achieve cost parity.

Alternately, a C-fuel supply unalterably short of demand may provide suitable boundary conditions for cost competiveness: that might be captured by multiplying the relative intrinsic utility $\ln (\beta b)$ with an externality factor $a$ measuring the impact of supply to demand ratio $\alpha$ on practical utility (no supply - no utility). There is certainly more than one way of doing this but any result must suffice three premises (see also ESI): first, for $\alpha=1$ the full base utility $\ln (\beta b)$ must be obtained; second, for $\alpha \rightarrow 0$ the factor $a$ respective utility must also approach zero and third, there is the requirement that $a \cdot \ln (\beta b) \leq \alpha$ but should still resemble $\alpha$, otherwise a residual utility at zero supply might ensue. 
A factor which basically suffices the former two premises is shown in equation 13a; however, the double logarithmic character results in too slow convergence and one logarithm must be offset by the exponent $e \approx 2.7183$ for compliance with the third requirement as by equation 13b. Equation $13 \mathrm{~b}$ virtually suffices all three premises but is evidently not usable if $\alpha$ is zero; hence the impact of supply and demand ratio $\alpha$ is expressed by the polynomial factor of equation 13c.

$a \ln (\beta b)=\left(\frac{\ln (2)}{\ln (2-\ln (\alpha))}\right) \ln (\beta b)$
$a \ln (\beta b)=\left(\frac{\ln (2)}{\ln (2-\ln (\alpha))}\right)^{e} \ln (\beta b)$

$a \ln (\beta b)=\left(1.0443 \alpha^{3}-1.1002 \alpha^{2}+1.0568 \alpha\right) \ln (\beta b)$

$(0 \leq \alpha \leq 1)$

For cost-parity the polynomial term of equation $13 \mathrm{c}$ must be at least equal to the reciprocal of the $\mathbb{E}_{96.0}$ values of relative $\mathrm{C}$-fuel cost, the critical supply to demand ratio $\alpha$ is numerically determined (ESI) and the results shown in table 5.

Table 5 The critical supply-to-demand ratio $\alpha$ for cost-neutral C-fuel substitution on basis of $\mathbb{E}_{96.0}$ values of table 3.

\begin{tabular}{|c|c|c|c|c|c|c|c|}
\hline & cH2 & $\begin{array}{c}\text { LH2 } \\
\text { (22 bar) }\end{array}$ & $\begin{array}{c}\text { LH2 } \\
\text { (350 bar) }\end{array}$ & LMH & LMH* & HMH & Li-ion \\
\hline $\mathbb{E}_{96.0}$ value of relative $\mathrm{C}$-fuel cost & 3.8 & 2.1 & 4.3 & 2.5 & 2.1 & 2.1 & 1.8 \\
\hline $\begin{array}{l}\text { Critical C-fuel supply to demand ratio } \alpha \\
\text { for cost parity (see also ESI) }\end{array}$ & $\begin{array}{c}0.325 \Rightarrow \\
\leq 33 \%\end{array}$ & $\begin{array}{c}0.614 \Rightarrow \\
\leq 61 \%\end{array}$ & $\begin{array}{l}0.280 \Rightarrow \\
\leq 28 \%\end{array}$ & $\begin{array}{l}0.521 \Rightarrow \\
\leq 52 \%\end{array}$ & $\begin{array}{l}0.614 \Rightarrow \\
\leq 61 \%\end{array}$ & $\begin{array}{l}0.614 \Rightarrow \\
\leq 61 \%\end{array}$ & $\begin{array}{c}0.697 \Rightarrow \\
\leq 70 \%\end{array}$ \\
\hline
\end{tabular}

Table 5 shows the required minimum C-fuel supply deficit for cost parity under the premise of a linear relation between cost and supply. That might be termed a embargo \& war economy scenario because these were the only situations in which such shortages occurred to date, never in a world market situation, not even in $1973 .{ }^{18}$ This reaches far out to oil shortage and surplus energy policies (shortage policy equals low end-consumer prices), the Petrodollar system and peak shift in the Peak Oil theory as it is related to a supply base which in turn is a reflects economic sensibility at a certain market price; yet the discussion of these further-reaching vital aspects merits a dedicated effort.

\section{Discussion}

At the outset of the discussion, a general delimitation of the underlying approach in to previous ones seems due: This work was initiated as an alternative draft to hitherto studies which despite excessive data volume are unable to provide even a faint idea of a compelling answer to the question whether hydrogen and other alternate electric energy technologies may become economically competitive to C-fuels one day or not (and if not what would be required for that). It might come as a surprise that this is for a good reason because they originate from a materialist, problem-fracturing mindset - at the latest by Descartes the hallmark of scientific problem-solving. This shows in the circumstances that a) C-fuel pathway(s) are treated as just one option among others and b) the ambition to determine (measure) the absolute cost of each option. The former does not do reality justice as Cfuels are the standard and the latter eliminates any possibility of an inner connecting principle, thus convergence in results. Having disassembled the problem in such manner, its reconstruction must rely on more or less imaginary cost tags for hooking up the fragments at the shortcoming of a severe problem of externalities, e.g. the entire well-to-tank part of well-to-wheel efficiency studies bases solely on estimates. Eventually, it comes down to speculations under which two elastic cost figures might meet and if that approach is followed through par force, it does not reveal the deus ex machina but leads into a quagmire of pointless detail. ${ }^{19}$ Technical-minded problem-solving is a fine 
servant within (largely) defined boundaries but a terrible master if applied to multi-layered, shadily defined issues. That is because of the inherent tendency to neglect essential information not directly visible in favour of the measurable. In that case inconclusiveness ensues, always an indicator for a misuse of the method because technical problems are convergent by their very nature: much effort for little gain, if any at all, is the common consequence.

In contrast, the present approach emphasises specific energy as an inner connecting principle: The line of argument originates from the fundamental notion that the world as we experience it is more or less visibly structured by the constraints of thermodynamics and with regard to energy technologies that causality is discernibly effective at the level of economics.

Energy can be expressed as product of pressure and volume - uncommon but valid - and specific energy can be thus reduced to a dimensionless figure if storage pressure and ensuing medium density are known. This is interpreted as the base advantage $b$ the utilization of this energy carrier offers. Wright's law is used as a vehicle for bringing the by itself rather meaningless advantage factor into effect in the reality of economics; in the process the thermodynamic advantage factor $b$ turns into its logarithmic pendant utility $\ln (b)$. This is viable on the grounds that Wright's law is a) empiric and $b$ ) if comparing two energy technologies in an ideal world (unrestricted access to labour and raw materials, equal production numbers, a steady market demand slightly ahead of production etc) any final disparity in cost must originate from a corresponding one in thermodynamic utility.

This first ideal baseline definition of utility $\ln (b)$ is subsequently adjusted for reality by introducing a non-specific efficiency factor $\beta$ for $\ln (\beta b)$ and its advantageously setting makes it possible to derive meaningful insight via the Bienaymé-Chebyshev inequality. The results are convergent and highlight immediately the relative final cost of C-fuel substitution respective the discrepancy which requires mitigation measures. That can be the basis for an informed decision-making process; independent of any criticism, this is the form of result towards which any solution for the issue should be structured.

The $96 \%$ probability values for relative final cost of the respective C-fuel substitution technologies range between 1.8 and 4.3 and it might be argued that these are just some fantasy figures from an idealized approach which coincidentally are in proportion but do not prove anything in an allegedly scientific objective manner since not measurable. Apart from the fact that arguing with a deadbeat solipsist materialistic point of view is a lazy fruitless endeavour, the logarithmic conception of utility and the relative structure of the statistical approach are exactly for the reason of coping with the issue of intangibles with expediency. Scope is less about an exhaustive truth but the definite largepicture baseline to relative cost and any further distorting externality from reality like a higher cost for a commodity due to limited availability can be still factored in if required. Yet this is incremental, not fundamental change: it is the structure of the approach which makes for the difference.

The outcome gets several empiric otherwise ill-explainable observations fundamentally right which shows most convincingly in the near-equal utility of 350 bar and 700 bar hydrogen utilization in tables 1 and 2, an observation already present in a well-to-wheel efficiency parameter study dating back to $2005 .{ }^{9}$ Whatever criticism there might be of the approach, the fundamental idea of an overall logarithmic relation at work appears vindicated in its own right. Within the concept of thermodynamic utility, a combination of high pressure and low density can negate any advantage a high specific energy may promise (axiomatic yet not so easy to show compellingly) and as a result, high-pressure hydrogen technologies score with distinction last on the utility scale. The principle works both ways as the metal hydride and Li-ion cases show and the economic sensibility of hydrogen as energy carrier seems generally related to low pressure and temperature technologies. The idea of thermodynamic utility makes by concept and in figures visible why the use of Hydralloy 
in the ThyssenKrupp Marine Systems submarines was sensible (development started more than 30 years ago $)^{20}$ and why Li-battery cars can compete in segments of the automobile market.

Since much of the C-fuel substitution narrative revolves around the automobile, the results of this study are applied to an exemplary comparison of the Skoda Superb, Tesla 3 and Toyota Mirai 2 production cost/net price. This is the only halfway sensible industrial-scale, application-based comparison of the kind possible to date and only entry models may be compared since representing the most cost competitive setup (for details see ESI). In sum, based on the $\mathbb{E}_{96.0}$ values of table 3 and the Skoda Superb as reference, the Tesla 3 U.S. production cost and EU net price (June 2020) are estimated to 27639 USD and 39793 EUR, respectively: the official net price is 39966.39 EUR.

Likewise, the (secret) true production cost and EU market price for the Toyota Mirai 2 are estimated to 77053 EUR and 113313 EUR, respectively. The June 2020 EU net price is listed with 63840 EUR but it is known that each Mirai sold at that price results in a secret 5-digit EUR respective USD loss to Toyota: that loss is thus figured to 49473 EUR at the prospective annual production of 30000 units.

Although the approach assesses the final relative cost separately from any political cost or issue of externalities, these immediately surface when facing the question how a disparity between 1.8 and 4.3 times the nowadays standard can be absorbed in an economy on a permanent basis. Considering that in many Western countries as of writing a suitable margin is claimed by taxes and duties, it does not require much imagination to develop a personal impression of the standard of living in a C-fuel substituted economy. However, whether the state is willing or actually can forgo that source of income on a permanent basis is an entirely different question and even if that were the case: What would the political and economic premises and consequences for that look like?

That fundamental issue of how to bridge the gap has been briefly addressed in this study by the examples of adjusted production numbers and C-fuel supply shortage but these are just two options out of a multitude of possibilities (e.g. deflation, doubling productivity and salaries, taking the spread just off the current living standard etc). Any answer branches quickly out into further questions about political cost of measures and reciprocations. Yet this paper is at its conclusion and it suffices to say that energy technologies represent the technical level to the strategic matter of energy policy and the results of this work comply better with that superstructure than those of hitherto ones. ${ }^{21}$

\section{Conclusion}

This work's line of argument originates from the fundamental idea that the world as we experience it is more or less visibly structured along the limiting causality of thermodynamics and in the case of energy technologies this becomes visibly effective at the level of economics. Introducing the concept of thermodynamic utility and using Wright's law as a carrier into the realm of economics, the model expresses its results relative to a C-fuel reference. The self-evident notion that it is unfavourable to use an energy technology which requires extreme temperatures and pressures is integral part of the approach. The model's forecast ability is founded on the Bienaymé-Chebyshev inequality and the cost-neutral substitution of C-fuels is an event very clearly below the $50 \%$ insight horizon of the model in all cases. The final figures suggest a relative marginal cost between 1.8 (Li-ion) to 4.3 (350 bar LH2) for substituting nowadays C-fuel standard - ideal case. The results comply reasonably well with hitherto findings and examples from reality. The base model may be extended for assessing economic and policy scenarios for mitigating cost disparity which has been shown by means of two examples. This study does by no means claim to be exhaustive but intends to mark out a starting position for a different, better way of dealing with the matter as it does not do any good to adhere to evidently non-convergent working concepts and hope that simply "more of it all" will eventually lead to a favourable conclusion of the matter. 


\section{Acknowledgements}

This paper spun-off from the author's main line of work done at FOTEC Forschungs- und Technologietransfer GmbH under European Space Agency grant 4000105330/12/NL/CLP and European Defence Agency contract A-1341-RT-GP which is gratefully acknowledged.

\section{Conflicts of Interest}

There are no conflicts of interest to declare.

\section{References}

1 T. P. Wright, J. Aeronaut. Sci., 1936, 3, 122-128.

2 Boston Consulting Group, Perspectives on Experience, Boston Consulting Group, 1972.

3 B. Nagy, J. D. Farmer, Q. M. Bui and J. E. Trancik, PLoS ONE, 2013, 8, e52669.

4 F. Lafond, A. G. Bailey, J. D. Bakker, D. Rebois, R. Zadourian, P. McSharry and J. D. Farmer, Technol. Forecast. Soc. Change, 2018, 128, 104-117.

5 G. Sandrock, K. Gross, G. Thomas, C. Jensen, D. Meeker and S. Takara, J. Alloys Compd., 2002, 330-332, 696-701.

6 P. Wang, X.-D. Kang and H.-M. Cheng, J. Appl. Phys., 2005, 98, 074905.

7 A. Zuettel, Demonstration eines Metallhydrid Speichers in einem mit Wasserstoff angetriebenen Pistenfahrzeug, Contract No. 1005, Final Report, Publication No. 250046, Swiss Federal Office of Energy SFOE, 2004.

8 B. D. MacDonald and A. M. Rowe, J. Power Sources, 2007, 174, 282-293.

9 N. Brinkman, M. Wang, T. Weber and T. Darlington, , DOI:10.2172/1218344.

10 H. Barthelemy, M. Weber and F. Barbier, Int. J. Hydrog. Energy, 2017, 42, 7254-7262.

11 E. Rothuizen, W. Mérida, M. Rokni and M. Wistoft-Ibsen, Int. J. Hydrog. Energy, 2013, 38, 4221-4231.

12 Lennie. Klebanoff, Hydrogen storage technology: materials and applications, Taylor \& Francis, Boca Raton, 2013.

13 K. Ohlig and L. Decker, Anchorage, Alaska, USA, 2014, pp. 1311-1317.

14 W. Luo, V. Stavila and L. E. Klebanoff, Int. J. Hydrog. Energy, 2012, 37, 6646-6652.

15 G. Petitpas, , DOI:10.2172/1466121.

16 F. A. L. Dullien, Porous media: fluid transport and pore structure, Academic Press, San Diego, 2nd ed., 1992.

17 R. H. Pawelke, The Master Key to the Problem of Reversible Chemical Hydrogen Storage is $12 \mathrm{~kJ}\left(\mathrm{~mol} \mathrm{H}_{2}\right)^{-1}, 2019$.

18 E. J. Mitchell, U.S. energy policy: a primer, American Enterprise Institute for Public Policy Research, Washington, 1974.

19 A. Reid, R. Nelson, S. Godwin, W. Weindorf, D. Rickeard, J.-F. Larivé, K. D. Rose, H. Maas, H. Hass, R. Edwards, L. Lonza, A. Krasenbrink, European Commission, Joint Research Centre and Institute for Energy and Transport, Well-to-tank report version $4.0 \mathrm{JEC}$ well-to-wheels analysis: well-to-wheels analysis of future automotive fuels and powertrains in the European context., Publications Office, Luxembourg, 2013.

20 A. Psoma and G. Sattler, J. Power Sources, 2002, 106, 381-383.

21 E. Luttwak, Strategy: the logic of war and peace, Belknap Press of Harvard University Press, Cambridge, Mass, Rev. and enl. ed., 2001. 